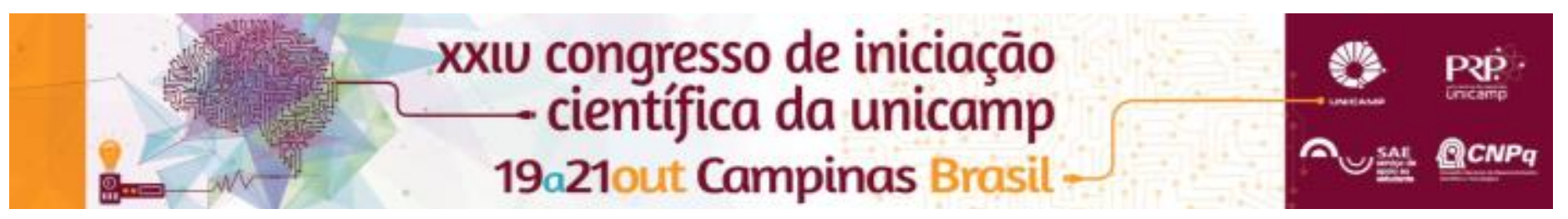

\title{
Architectures for Optical Networks SLICE
}

\section{Wellington Renan Gonçalves*}

\begin{abstract}
The aim of this work is to study the new optical network architectures, in special, the type SLICE (Spectrum-Sliced Elastic Optical Path) and its components. Moreover, to build and to run simulations of this type of architecture in MATLAB and OptiSystem 7.0 tools evaluating their results.
\end{abstract}

Key words:

elastic optical networks, NG-ROADM, telecommunicatioins systems.

\section{Introduction}

In new generation of optical networks type SLICE (Spectrum-Sliced Elastic Optical Path), the optical spectrum is treated as a continuing resource, allowing greater compression and efficiency in the use of it. Basically, the optical path can be allocated to any continuous frequency range and must be separated from other optical paths by a guard band called FBG (Filter Guard Band). The new optical transceivers use the OFDM (Orthogonal Frequency-Division Multiplexing) modulation that enables data to be sent on orthogonal subcarriers and the subcarrier signals of each pair extend up to the neighboring spectrum without any interference among them. With the introduction of these news elements into to the optical network and its new form of spectrum use, new concepts of routing, traffic management, restoration and protection have to be researched and developed. The aim of this work is to study these new optical network architectures, in special, the architecture type SLICE and its components. Moreover, to build and to run simulations of this type of architecture in MATLAB and OptiSystem 7.0 tools evaluating their results.

\section{Results and Discussion}

The Figure 1 shows an example of the power management in SLICE optical networks using ring topology and EDFAs (Erbium-Doped Fiber Amplifiers). The aim of the power management is to ensure that enough power reach the optical receiver to maintain a reliable performance throughout the entire system lifetime. In such example, EDFAs are inserted before the inputs of the multiplexers/demultiplexers in each node to offset the losses of the previous node. Analyzing the bit error rate through the BER Analyser, in Figure 2, we can see the improvement in signal quality when compared to a system that does not use EDFAs on each link.

\section{Conclusions}

In order to better understand the architecture of new optical networks type SLICE, a theoretical study of optical communications, as well a simulation management system using OptiSystem 7.0 tool were conducted.

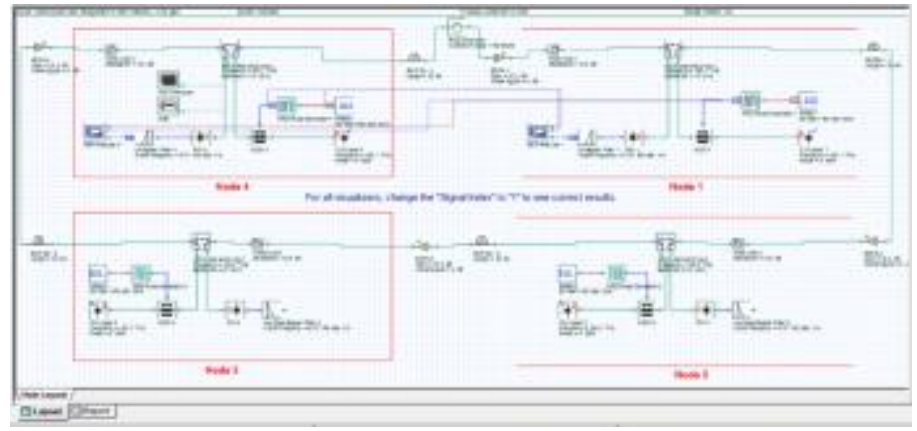

Figure 1. Power management for optical networks type SLICE.

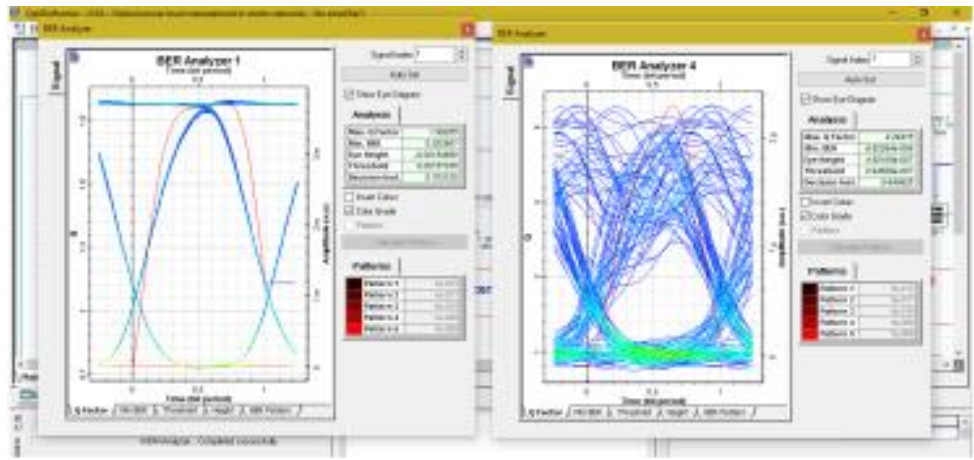

Figure 2. Signal quality.

\section{Acknowledgement}

Thanks to Professor Luis Fernando de Avila by knowledge transference during the Project, and PIBITI/FAEPEXISAE by the financial support.

\footnotetext{
${ }^{1}$ Govind P. Agrawal; tradução José Rodolfo Souza: "Sistemas de comunicação por fibra óptica". 4. Ed. - Rio de Janeiro: Elsevier, 2014.

${ }^{2}$ Optiwave Systems Inc. "Fiber-Optic Communications Systems, Fourth Edition by Govind P. Agrawal. Book Examples”. Consultado em 19 de Janeiro de 2016. <http://optiwave.com/resources/academia/download-area/>
} 